\title{
Experimental Procedure for the Determination of Cavity Parameters*
}

\author{
Amnon Yarivi and Fred D. Clapp \\ Electronics Research Laboratory, University of California, Berkeley, California
}

(Received December 22, 1958; and in final form, May 28, 1959)

\begin{abstract}
A new experimental procedure for the determination of cavity parameters is described. The procedure follows the basic steps described by Slater. In this method one measures the frequencies at which the voltage standing wave ratio at the input to the cavity reaches a predetermined value. The knowledge of these frequencies coupled with a determination of the state of coupling to the cavity yields the necessary information. An exposition of some pertinent theoretical points is followed by a detailed description of the experimental procedure.
\end{abstract}

\section{INTRODUCTION}

A NEW experimental procedure for the measurement of the parameters of resonant microwave cavities is described. The new features are a dynamic determination of the frequency deviation $\Delta f$ at which the cavity causes a predetermined mismatch, and a dynamic determination of the coupling condition of the cavity.

The combination of a swept frequency source and a null detection minimizes the importance of the frequency stability and renders the knowledge of the response law of the receiver (linear, square law) unnecessary.

The procedure has been used by the authors in electron paramagnetic resonance work where the microwave bridge used in the electronic resonance detection was the same as the one used for the cavity measurements. This made it possible to recalibrate the cavity after each change in sample or temperature without disrupting the system.

\section{THEORETICAL INTRODUCTION}

\section{A. Measurement of Voltage Standing Wave Ratio}

The experimental procedure described below is based entirely on dynamic measurements of voltage standing wave ratio (VSWR) and frequency differences. The VSWR is measured by means of a calibrated probe. ${ }^{1}$ The probe is moved along the wave guide and its penetration is varied until a match is obtained. The VSWR is equal to that caused by the probe when placed in a line terminated by a matched load. To justify this procedure we may invoke the theory of transmission lines where it can be shown that a given shunt reactance when placed in the proper place along the line will match out a VSWR equal to the one set up by the same reactance when placed in front of a matched termination. The VSWR as a function of probe penetration was measured in a seperate experiment and recorded on a calibration chart.

* This research was supported by the U. S. Air Force through the Air Force Office of Scientific Research of the Air Research and Development Command, under Contract No. AF 49(638)-102.

$\dagger$ Present address: Bell Telephone Laboratories, Murray Hill, New Jersey.

${ }^{1}$ G. B. Collins, Microwave Magnetrons, Radiation Laboratory Series, (McGraw-Hill Book Company, Inc., New York, 1958), p. 708.

\section{B. Coupling Condition of the Cavity}

The overcoupled cavity, the critically coupled cavity, and the undercoupled cavity are characterized by the ratio of the external " $Q$ " to the internal " $Q$ "'; this ratio is denoted by $a$.

$$
a=\text { external } Q / \text { cavity } Q=Q_{x} / Q_{a} .
$$

For the three cases of coupling listed in the foregoing $a<1, a=1$, and $a>1$, respectively.

A measurement of the VSWR at resonance yields the following information ${ }^{2}$ :

$$
\begin{array}{ll}
(\mathrm{VSWR})_{\mathrm{res}}=1 / a & \text { for an overcoupled cavity, } \\
(\mathrm{VSWR})_{\mathrm{res}}=1 & \text { for a critically coupled cavity, } \\
(\mathrm{VSWR})_{\mathrm{res}}=a & \text { for an undercoupled cavity. }
\end{array}
$$

Since the overcoupled and undercoupled cavities are indistinguishable in terms of VSWR measurements alone, a separate test must be used to determine the coupling condition. In this experiment, where the frequency is swept, we make use of the different behavior of the three types of cavities when the loci of their reflection coefficients are

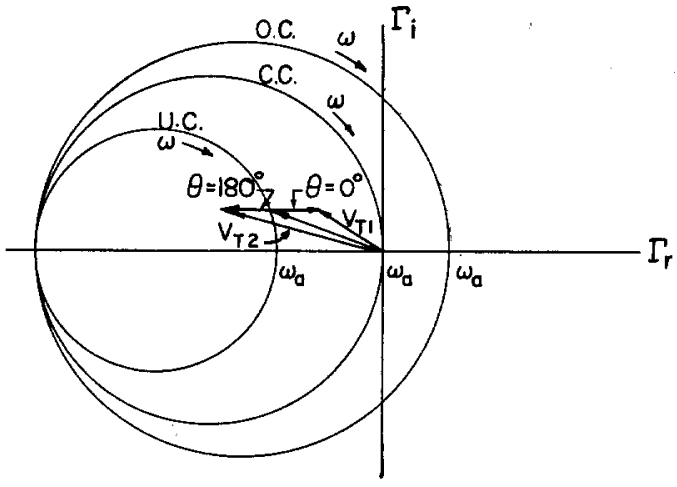

Fig. 1. The loci of the complex reflection coefficient $\Gamma$ of overcoupled, undercoupled, and critically coupled cavities. The loci are plotted in the complex $\Gamma$ plane with $\omega$ as the parameter. The vector construction shows the effect of adding to the voltage refiected from an undercoupled cavity, at an arbitrary frequency, two external voltages of opposite phases to each other.

2 J. Slater, Microwave Electronics (D. Van Nostrand Company, Inc., Princeton, New Jersey, 1950), Chap. V. 
plotted in the complex reflection coefficient plane $(\Gamma)$ with radian frequency $\omega$ as the parameter. ${ }^{2}$ A small external voltage $V_{2}$ is added to that reflected from the cavity. The behavior of the total voltage, as the phase of $V_{2}$ is varied and the frequency swept, can be easily used to distinguish between the overcoupled and undercoupled cavity. Figure 1 shows the total voltage $V_{\text {ref1 } 1}+V_{2}$ for an undercoupled cavity at one frequency for two opposite phases of $V_{2}$. Performing a series of similar constructions at different frequencies for an undercoupled and overcoupled cavities and plotting the magnitude of the total voltage as a function of frequency yields curves similar to these of Fig. 2. The marked difference between the overcoupled and undercoupled cavities is due to the reversal in sign of the real part of the reflection coefficient of an overcoupled cavity as contrasted with the undercoupled cavity which has one sign for all frequencies.

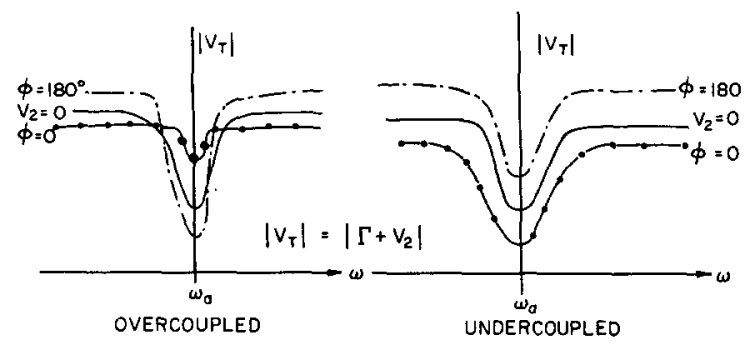

(a)

(b)

FIG. 2 (a) For an overcoupled cavity, the absolute value of the sum (voltage reflected from the cavity + an external voltage) is shown as a function of frequency for different phases of the external voltage and for no external voltage. (b) For an undercoupled cavity, the absolute value of the sum (voltage reflected from the cavity + an external voltage) is shown as a function of frequency for different phases of the external voltage and for no external voltage.

\section{The VSWR of a Cavity}

According to. Slater the normalized input admittance at a single port cavity taken at the position of the detuned short is ${ }^{2}$

$$
y=\frac{Y}{Y_{0}}=\frac{Q_{x}}{Q_{a}}+j 2 Q_{x} \frac{f-f_{a}}{f_{\alpha}},
$$

where $f_{a}=$ resonant frequency of the $a$ th mode, $Y_{0}=$ characteristic admittance of input guide, and $Y=$ input admittance. If we define

$$
S=Q_{x} \frac{2\left(f-f_{a}\right)}{f_{\alpha}},
$$

we have

$$
\text { VSWR }=\frac{1+|\Gamma|}{1-|\Gamma|}=\frac{1+\frac{\left[\left(1-a^{2}-S^{2}\right)^{2}+4 S^{2}\right]^{\frac{1}{2}}}{(1+a)^{2}+S^{2}}}{1-\frac{\left[\left(1-a^{2}-S^{2}\right)^{2}+4 S^{2}\right]^{2}}{(1+a)^{2}+S^{2}}}
$$

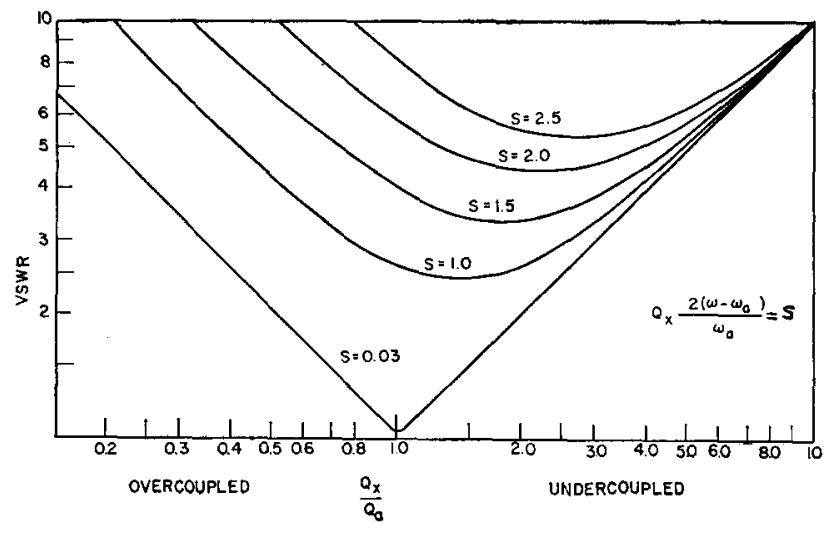

Fig.; . Universal curves giving the voltage standing wave ratio of a cavity as a function of the coupling factor $Q_{x} / Q_{a}$ with $S=Q_{x}\left[2\left(\omega-\omega_{a}\right)\right] / \omega_{a}$ as a parameter.

The VSWR is plotted in Fig. 3 as a function of $a=Q_{x} / Q_{a}$ with $S$ as a parameter.

\section{STEP-BY-STEP PROCEDURE FOR DETERMINATION OF CAVITY PARAMETERS}

\section{Experimental Setup}

The experimental setup is shown in Fig. 4. The klystron frequency is swept by applying a sawtooth voltage to the repeller. The same voltage is applied to the horizontal plates of the oscilloscope. The addition of an external voltage of variable phase and amplitude to the voltage reflected from the cavity is done by means of a slide screw turner placed in the magic- $T$ arm opposite to the cavity arm. The total voltage is detected and applied to the vertical deflection plates of the oscilloscope.

\section{Step 1. Measurement of the Cavity Resonant Frequency}

When the two probes are pulled out the oscilloscope displays the power reflected from the cavity as a function of frequency, the observed dip occurring at $f_{a}$, the cavity resonant frequency. This frequency can be read on the calibrated wave meter when the meter dip is made to coincide with that of the cavity.

\section{Step 2. Measurement of $Q_{x} / Q_{a}$}

With probe 1 pulled out, the penetration of probe 2 is increased and its position along the wave guide varied

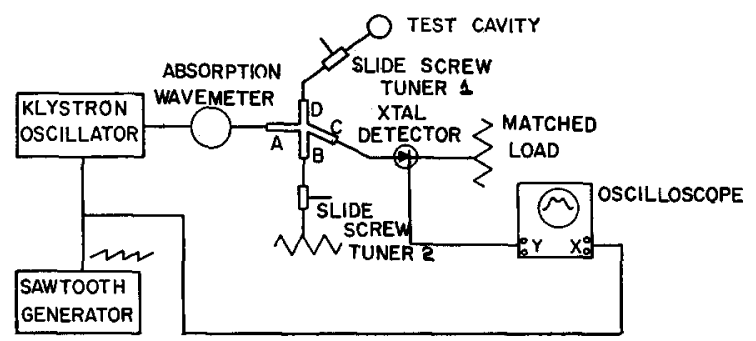

FIG. 4. The experimental setup. 


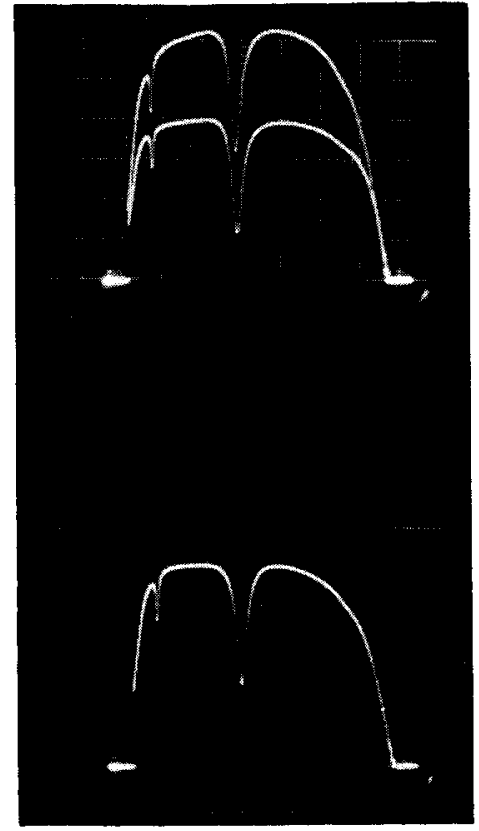

FIG. 5. Undercoupled cavity. An oscillogram of the variation of the absolute value of the sum (reflected voltage + external voltage) as a function of frequency for two opposite phases of the external voltage. The lower trace corresponds to the case of no external voltage.

until a perfect match is obtained. The match condition is indicated by a dip of the reflected power all the way to the zero level on the oscilloscope. The VSWR is read off the calibration chart. The value of the VSWR thus obtained is equal, according to the discussion of Sec. $B$ to $Q_{x} / Q_{\alpha}$ or $Q_{a} / Q_{x}$ depending on the coupling condition.

\section{Step 3. Determination of the Coupling Condition}

To determine $Q_{x} / Q_{a}$ we have to know whether the cavity is overcoupled or undercoupled. This determination uses the procedure described in Sec. B. The voltage $V_{2}$ is added by means of probe 2 which when inserted causes a reflection from the otherwise matched wave guide. The phase of $V_{2}$ is controlled by moving the probe along the wave guide. If the "shoulders" and "valley" of the resonance oscillogram move up and down simultaneously as probe 2 is moved back and forth along the wave guide the cavity is undercoupled and $Q_{x} / Q_{a}=$ VSWR. If the "shoulders" and "valley" move in opposite directions the cavity is overcoupled and $Q_{x} / Q_{a}=1 /$ VSWR. Actual oscillograms of this test performed on an undercoupled and overcoupled cavities are shown in Figs. 5 and 6.

\section{Step 4. Measurement of the External " $Q$ "}

If at a known frequency deviation $\left(f-f_{a}\right)$, a cavity of coupling ratio $Q_{x} / Q_{a}$ causes a VSWR $\rho_{2}$, its external " $Q$ " $Q_{x}$ is uniquely determined. This statement can be verified by means of Eq. (2). The exploitation of this idea in our experiment consists of introducing a known VSWR into the cavity input wave guide by means of the calibrated probe and then measuring the frequency at which the cavity causes an equal VSWR. At this frequency we can find, by moving the probe along the wave guide, a series of points separated by half a guide wavelength at which the two reflections interfere destructively to yield a matched condition. The experimental procedure is as follows: Fig. 3 is entered at a position on the abscissa corresponding to the $Q_{x} / Q_{a}$ ratio of the cavity; the corresponding VSWR is read on any one of the curves. Having selected a particular curve, $S$ is determined. Probe 1 is inserted to a depth corresponding to the VSWR and is moved along the wave guide until, at some frequency, a match obtains. The match is indicated by a dip to the zero power level on the oscilloscope. The match can be made to occur at either one of two frequencies $f_{u}$ and $f_{l}$ by merely moving the probe along the wave guide. The two frequencies are symmetrically situated about $f_{a}$, i.e.,

$$
f_{u}-f_{a}=f_{a}-f_{l} .
$$

The quantity $\left(f_{n}-f_{l}\right)$ is determined by means of the resonant wave meter. Since the quantity $S$ which is equal to $Q_{x}\left(f_{u}-f_{l}\right) / f$ is known, $Q_{x}$ can be calculated from the relation

$$
Q_{x}=S f_{\alpha} /\left(f_{u}-f_{l}\right) .
$$

$f_{a}, Q_{x} / Q_{a}$, and $Q_{x}$ have thus been found.

\section{Miscellaneous Remarks}

(1) A backward wave oscillator with a swept beam voltage can be used, instead of a klystron, for the signal source.

(2) If no resonance is observed the cause is either failure to cover the resonant frequency during the sweep, or that the cavity is too overcoupled or undercoupled.

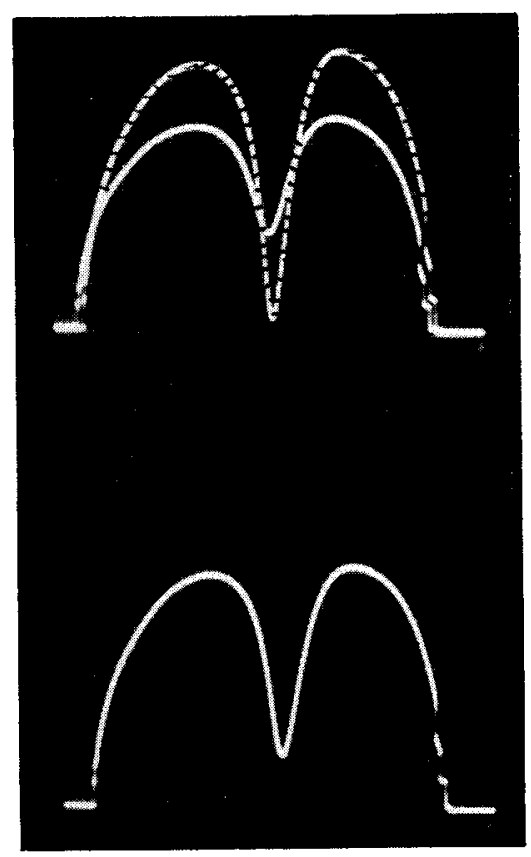

FIG. 6. Overcoupled cavity. An oscillogram of the variation of the absolute value of the sum (reflected voltage + external voltage) as a function of frequency for two opposite phases of the external voltage. The lower trace corresponds to the case of no external voltage. 
(3) The accuracy of the procedure is limited by the resolution of the wave meter used to determine $\left(f_{u}-f_{l}\right)$. It is estimated as $\pm 15 \%$ for typical low mode number, $X$-band cavities $(Q \simeq 10000)$ when using the best available commercial wave meters. For larger accuracy or at higher frequencies a more accurate method of frequency measurement must be used. One method found satisfactory utilizes frequency markers which are derived by mixing the swept frequency signal with a stable constant frequency signal coming from another source (stabilized klystron or harmonic generator and multiplier) and feeding the difference frequency into an FM receiver. The accuracy was estimated as $\pm 5 \%$ at $20 \mathrm{kMc}$.

(4) Dr. J. P. Gordon of the Bell Telephone Laboratories suggested that probe 2 can be eliminated and its role taken over by probe 1 in the determination of the coupling condition. The suggestion was tried out successfully by Dr. C. F. Hempsted of these Laboratories.

\section{ACKNOWLEDGMENTS}

The authors take pleasure in thanking Professor A. F. Kip and Professor J. R. Whinnery for helpful discussions on this subject.

\title{
Rapid Insertion Device for Coaxial Attenuators
}

\author{
A. Y. Rumfelt and R. J. Como \\ Radio Standards Laboratory, National Bureau of Standards, Boulder, Colorado
}

(Received May 4, 1959; and in final form, June 4, 1959)

\begin{abstract}
A rapid insertion device for coaxial attenuators is described. This device is used as an alignment aid when making insertion loss measurements of fixed coaxial attenuators at frequencies up to $6 \mathrm{kMc}$. It provides a quick connect and disconnect feature, maintains axial alignment of the connectors during joining and separation, and reduces cable flexure. The improved repeatability and accuracy of insertion loss measurements is shown by comparative data with different alignment techniques.
\end{abstract}

$I^{\perp}$ $\mathrm{N}$ measuring the insertion loss of a coaxial attenuator, separation of a pair of mating connectors and joining of two pairs of mating connectors are required in order to insert the attenuator. Hence, considerable time is used in separating and joining connectors, especially in systems using threaded connectors, such as type N. This permits random variations in the output level during the insertion loss measurements due to system instability. Also, such fluctuations may be partly due to effects associated with flexing of the cables especially at frequencies above 3000 Mc. ${ }^{1}$

The insertion device, shown in Fig. 1, was designed to perform the following functions: (1) provide a quick connect and disconnect feature, (2) maintain axial alignment of connectors during joining and separation, and (3) reduce cable flexure. The removal of the threads of the system

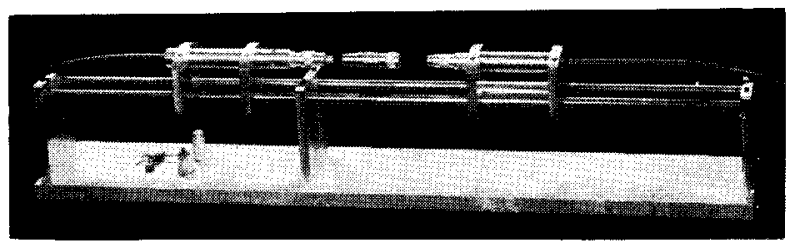

Fig. 1. Rapid insertion device.

${ }^{1}$ J. R. Hannon, "Braid Design for Coaxial Cables for Frequencies above $3000 \mathrm{Mc} / \mathrm{s}$," Navships 900,191 , Bureau of Ships, Navy Department, Washington 25, D. C., October 21, 1955. connectors where insertion occurs allows quick opening of the transmission line system and quick insertion of the attenuator. This reduces the effects of medium-time instability of the system. The sliding mechanism maintains the axial alignment of the connectors, which during separation and joining becomes uncertain when the threads are removed. The metal sleeves attached to the connectors hold the cable eliminating its flexure near the connector and thereby reducing difficulties from this source. The modification detals of the type $\mathrm{N}$ connectors and the construction details of the cable sleeves are shown in Fig. 2.

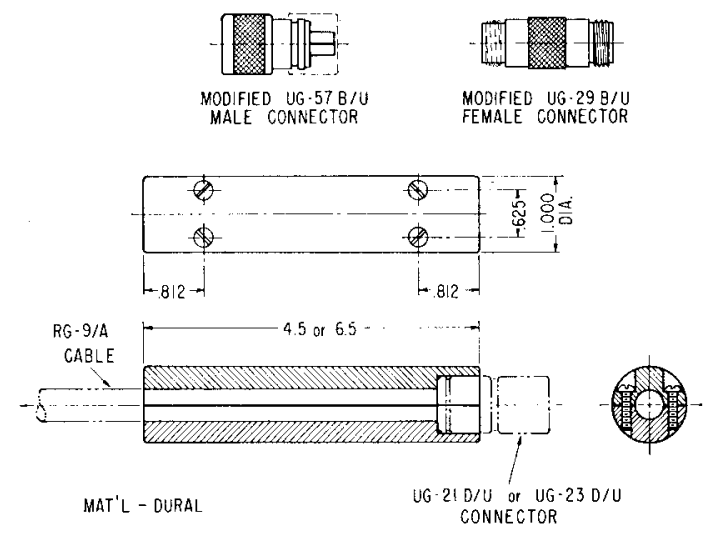

FIG. 2. Construction details of the cable sleeves and modified type $\mathrm{N}$ connectors. 